Artículo

\title{
Fases de desarrollo y propagación de ecotipos destacados de Tithonia diversifolia (Hemsl.) A. Gray
}

Julián Esteban Rivera-Herrera $\mathrm{a}^{*}$

Tomás Ruíz-Vásquez ${ }^{\text {b }}$

Julián Chará-Orozco ${ }^{\text {a }}$

Juan Florencio Gómez-Leyva ${ }^{c}$

Rolando Barahona-Rosales ${ }^{\mathrm{d}}$

${ }^{a}$ Centro para la Investigación en Sistemas Sostenibles de Producción Agropecuaria CIPAV. Carrera 25 \# 6 - 62 Cali, Colombia.

${ }^{\mathrm{b}}$ Instituto de Ciencia Animal, San José de las Lajas. La Habana, Cuba.

c Instituto Tecnológico de Tlajomulco, TecNM. Laboratorio de Biología Molecular. Jalisco, México.

${ }^{\mathrm{d}}$ Universidad Nacional de Colombia, Sede Medellín, Colombia.

*Autor de correspondencia: jerivera@fun.cipav.org.co

\section{Resumen:}

La propagación eficiente de especies vegetales con alto potencial alimenticio juega un papel fundamental en su adopción y utilización por parte de los productores. Es importante, por tanto, desarrollar métodos económicos y rápidos para el establecimiento exitoso de sistemas más productivos. Con el objetivo de conocer algunos aspectos reproductivos de diferentes genotipos destacados de $T$. diversifolia y así favorecer su propagación y mejorar su uso en la alimentación animal, se evaluó su potencial de germinación, producción de semilla y duración del ciclo reproductivo. Se usaron 42 parcelas en un diseño de bloques completos al azar con siete genotipos de T. diversifolia y dos niveles (sin y con fertilización). Los genotipos tuvieron diferencias en todas las variables agronómicas y de producción de semilla a excepción del tiempo de secado de aquenios y en el porcentaje de semillas rudimentarias $(P<0.01)$. Con relación a la 
producción de semilla sexual, la fertilización tuvo efecto significativo $(P<0.05)$ y en general incrementó el tiempo de las fases evaluadas. La mayor producción de semilla se dio en los genotipos 7 y 5 y el porcentaje de germinación tuvo diferencias significativas entre genotipos y entre los tratamientos pre-germinativos $(P=0.0001)$ con mayores porcentajes en los genotipos 3 y 6 . Se concluye que a pesar de la baja viabilidad de la semilla sexual de $T$. diversifolia, existen genotipos con un mayor potencial de producción de semilla y porcentaje de germinación, parámetros que pueden ser mejorados por medio de tratamientos pre-germinativos y el uso de fertilizantes.

Palabras clave: Fase reproductiva, Germinación, Propagación vegetal, Semilla sexual, Viabilidad de la semilla.

Recibido: 26/06/2020

Aceptado:28/10/2020

\section{Introducción}

La inclusión de plantas forrajeras altamente proteicas y con bajo contenido de fibra en la dieta de bovinos, resulta en un incremento de la calidad de la biomasa forrajera, que, a su vez, se traduce en un aumento de la producción de leche y carne ${ }^{(1,2)}$. Por esta razón, una adecuada y eficiente propagación debe ser un objetivo de estudio para mejorar su utilización.

Tithonia diversifolia (Hemsl.) A. Gray es una arbustiva de la familia Asteraceae que, por su facultad de adaptación a múltiples condiciones ambientales, edáficas y de manejo, capacidad de rebrote y rápido crecimiento, y alto valor y aporte nutricional, ha demostrado su potencial para la alimentación animal ${ }^{(3,4,5)}$. T. diversifolia puede reproducirse tanto por semilla gámica como por semilla asexual, confiriéndole gran capacidad de reproducción y colonización de nuevos hábitats ${ }^{(6,7)}$. Esta especie florece y produce semillas durante todo el año especialmente en los meses de octubre y noviembre, aunque por condiciones ambientales puede ser de floración anual ${ }^{(8,9)}$. Sin embargo, algunas investigaciones han reportado porcentajes de germinación menores del $30 \%$ en condiciones naturales ${ }^{(7,10)}$.

Aunque observaciones de campo indican que T. diversifolia tiene una gran capacidad para crecer clonalmente $^{(6)}$, en la actualidad se sabe que material proveniente de semilla sexual puede favorecer el desarrollo de sistemas radiculares más extensos, plantas más vigorosas, mayor persistencia de los cultivos y recuperación más rápida después del corte o pastoreo ${ }^{(11)}$. Sin embargo, aún es difícil alcanzar material seminal de buena calidad, y adicionalmente, dentro de esta especie existen genotipos con diferentes capacidades de germinación ${ }^{(11,12)}$. Al estudiar el desarrollo de la microsporogénesis, se reconocen 
anormalidades cromosómicas en $32 \%$ de células en metafase I y anafase I, identificando cromosomas rezagados. Por otro lado, las razones de la esterilidad del polen en Tithonia podrían converger en las irregularidades observadas en su división meiótica ${ }^{(13)}$.

Con el objetivo de conocer algunos aspectos reproductivos de genotipos destacados de $T$. diversifolia en Colombia y así favorecer su propagación y mejor uso hacia la alimentación animal, se evaluó el potencial de germinación, producción de semillas viables y duración de las fases de crecimiento.

\section{Material y métodos}

\section{Genotipos evaluados}

Se evaluaron siete genotipos destacados para la alimentación animal de $T$. diversifolia identificados previamente en un análisis de diversidad genética ${ }^{(14)}$ y colectados en diferentes sitios de Colombia.

\section{Localización}

Las mediciones se desarrollaron en parcelas experimentales localizadas en municipio de San Luis de Cubarral (Meta, Colombia), a 347'21.43"N, 7349'15.93"O y a una altura sobre el nivel del mar de $530 \mathrm{~m}$. El sitio cuenta con una precipitación media anual de $4,100 \mathrm{~mm}$, una temperatura promedio de $24.8^{\circ} \mathrm{C}$ y se ubica en la zona de vida de bosque húmedo tropical $(\mathrm{bh}-\mathrm{T})^{(15)}$.

\section{Medición de condiciones ambientales}

Durante todo el periodo de muestreo las variables ambientales de precipitación $(\mathrm{mm})$, temperatura $\left({ }^{\circ} \mathrm{C}\right)$, humedad relativa $(\%)$, radiación solar $\left(\mathrm{W} / \mathrm{m}^{2}\right)$, punto de rocío $\left({ }^{\circ} \mathrm{C}\right)$, velocidad del viento $(\mathrm{m} / \mathrm{s})$ e índice THSW (sensación térmica a causa del viento, la humedad relativa), la irradiancia (radiación solar instantánea) y la temperatura $\left({ }^{\circ} \mathrm{C}\right)$ fueron monitoreadas por medio de una estación meteorológica Vantage Pro $2^{\mathrm{TM}}$ (Davis $®$ ).

\section{Análisis de suelos}

Las variables químicas y físicas de $\mathrm{pH}, \mathrm{C} . \mathrm{E}(\mathrm{dS} / \mathrm{m})$, capacidad de campo (\%), punto de marchitez permanente $(\%)$, densidad aparente $(\mathrm{g} / \mathrm{cc})$, carbono orgánico $(\%)$, materia orgánica $(\%)$, textura, potasio intercambiable $(\mathrm{mg} / \mathrm{kg})$, calcio intercambiable $(\mathrm{mg} / \mathrm{kg})$, magnesio intercambiable $(\mathrm{mg} / \mathrm{kg})$, sodio intercambiable $(\mathrm{mg} / \mathrm{kg})$, acidez intercambiable $(\mathrm{mg} / \mathrm{kg})$, hierro (mg/kg), manganeso ( $\mathrm{mg} / \mathrm{kg})$, cobre (mg/kg), zinc $(\mathrm{mg} / \mathrm{kg})$, boro $(\mathrm{mg} / \mathrm{kg})$, fósforo $(\mathrm{mg} / \mathrm{kg})$, azufre $(\mathrm{mg} / \mathrm{kg})$ y la C.I.C.E. (meq/100 $\mathrm{g}$ ), fueron determinadas en el suelo donde se encontraron las parcelas experimentales. 


\section{Variables de crecimiento y reproducción}

Las variables de duración de la fase vegetativa (días), duración de la fase reproductiva (días), duración del secado de aquenios (días), fase de floración (días), cabezuelas por plantas (\#), semillas por cabezuela (\#), semillas por planta (\#), semillas llenas (\%), semillas vacías (\%) y semillas rudimentarias (\%), fueron medidas en los siete genotipos evaluados. Las duración de la fase vegetativa se determinó desde el momento del corte de uniformización en el que las plantas se podaron a $15 \mathrm{~cm}$ de altura hasta el inicio de la floración de más del $50 \%$ de los individuos que conformaban las parcelas experimentales; la duración de la fase reproductiva fue desde el momento de la aparición de botones florales hasta la caída de pétalos en el $50 \%$ de las plantas; la duración de secado de aquenios fue desde la caída de pétalos en las plantas hasta lograr un color marrón oscuro de las cabezuelas, y la fase de floración fue la sumatoria de las fases vegetativa, reproductiva y secado de aquenios. Las variables de cabezuelas por plantas (\#), semillas por cabezuela (\#), semillas por planta (\#), semillas llenas (\%), semillas vacías (\%) y semillas rudimentarias (\%), se midieron de forma manual y en cinco plantas elegidas al azar de cada una de las parcelas experimentales a partir del cálculo de tamaño de la muestra, asumiendo un error máximo de estimación aceptado y nivel de confianza del $10 \%$. El cultivo evaluado tenía una edad de 13 meses y las mediciones se realizaron en la época de lluvias durante el segundo periodo del año 2019.

\section{Germinación de la semilla sexual}

Se evaluaron dos tratamientos pre-germinativos y un tratamiento sin proceso previo (Trat 1). Los tratamientos pre-germinativos fueron: agua a $80^{\circ} \mathrm{C}$ durante $10 \mathrm{~min}$ (Trat 2$)^{(16,17,18)}$, y ácido sulfúrico al $50 \%\left(\mathrm{H}_{2} \mathrm{SO}_{4}\right)$ durante $5 \mathrm{~min}(\text { Trat } 3)^{(19)}$. La semilla estuvo almacenada durante cuatro meses después de ser colectada con el objetivo de disminuir la latencia fisiológica de la semilla ${ }^{(17,20)}$, y la germinación se evaluó en macetas durante 25 días usando 50 semillas por sitio.

\section{Diseño experimental y análisis de la información}

Los siete genotipos de $T$. diversifolia se establecieron en parcelas experimentales en un diseño de bloques completos al azar con dos factores (el genotipo y el nivel de fertilización). Cada material contó con tres repeticiones las cuales estuvieron conformadas por 36 plantas cada una $(0.8 \mathrm{~m}$ x $0.8 \mathrm{~m})$ y dos niveles de fertilización (cero fertilización y fertilización de acuerdo con la extracción de nutrientes a los 40 días de crecimiento). Según evaluaciones, plantas de T. diversifolia de 40 días extraen $8.26 \mathrm{~g}$ de nitrógeno, $4.3 \mathrm{~g}$ de potasio y $1.07 \mathrm{~g}$ de fósforo ${ }^{(21)}$. Esta cantidad de nutrientes fue aplicada fertilizando con urea (46\% de N), fosfato biamónico (DAP) $\left(\left(\mathrm{NH}_{4}\right) 2 \mathrm{HPO}_{4}\right)(46 \%$ de $\mathrm{P}_{2} \mathrm{O}_{5}, 18 \%$ de $\left.\mathrm{N}\right)$ y cloruro de potasio $\left(\mathrm{KCl}, \mathrm{K}_{2} \mathrm{O}\right.$ del 60 al $63 \%$ y $\mathrm{Cl}$ del 45 al $47 \%$ ) a razón de16.22, 2.15 y $4.89 \mathrm{~g} /$ planta de urea, DAP y $\mathrm{KCl}$, respectivamente. Las 
evaluaciones de germinación igualmente se analizaron bajo el mismo diseño experimental, asignando macetas de acuerdo con las parcelas dispuestas en campo. El modelo matemático del diseño experimental fue:

$\mathrm{yklj}=\mu+\alpha \mathrm{k}+\gamma \mathrm{l}+\xi \mathrm{kl}+\xi \mathrm{kl}+\beta \mathrm{j}+\mathrm{Qklj}$

Donde:

yklj = Observación en la unidad experimental de la variable a evaluar;

$\boldsymbol{\mu}=$ es la media del efecto general;

$\boldsymbol{\alpha} \mathbf{k}=$ efecto del factor $\mathrm{k}$ (materiales colectados 1, 2, 3...7);

$\gamma \mathbf{l}=$ efecto del factor 1 (nivel de fertilización $1,2 \ldots$ );

$\boldsymbol{\beta} \mathbf{j}=$ efecto del bloque $\mathbf{j}$;

$\boldsymbol{\xi} \mathbf{k l}=$ interacción de los dos factores;

$\mathbf{q k l j}$ = valor aleatorio, error experimental de la unidad experimental lkj.

Todos los análisis se realizaron en la herramienta RStudio usando la librería "agricolae"(22). Para los análisis se evaluó la normalidad, homogeneidad de la varianza y la aditividad, además, cuando fue identificada diferencia entre las medias se utilizó la prueba de contraste de Tukey, con un nivel de significancia de 0.05. Finalmente, en las variables de fase reproductiva y semillas llenas, cuando los grupos de datos no cumplieron con las condiciones para un análisis paramétrico, se usó la prueba de Kruskal-Wallis y Mann-Whitney para las comparaciones.

\section{Resultados}

\section{Condiciones ambientales}

La Figura 1 muestra las condiciones ambientales observadas durante el periodo de evaluación. Según los registros obtenidos en el sitio de experimentación, la temperatura promedio fue de $23.9 \pm 1.2{ }^{\circ} \mathrm{C}$, la humedad relativa fue de $83.4 \pm 5 \%$, el punto de rocío promedio fue $20.8 \pm 0.6{ }^{\circ} \mathrm{C}$, la velocidad del viento fue de $0.7 \pm 0.2 \mathrm{~m} / \mathrm{seg}$, el índice THSW promedio fue $26.7 \pm 2.1^{\circ} \mathrm{C}$, la radiación solar $147 \pm 53.3 \mathrm{~W} / \mathrm{m}^{2}$ y la precipitación acumulada fue de $556.2 \mathrm{~mm}$. 
Figura 1: Condiciones ambientales observadas durante el periodo de experimentación
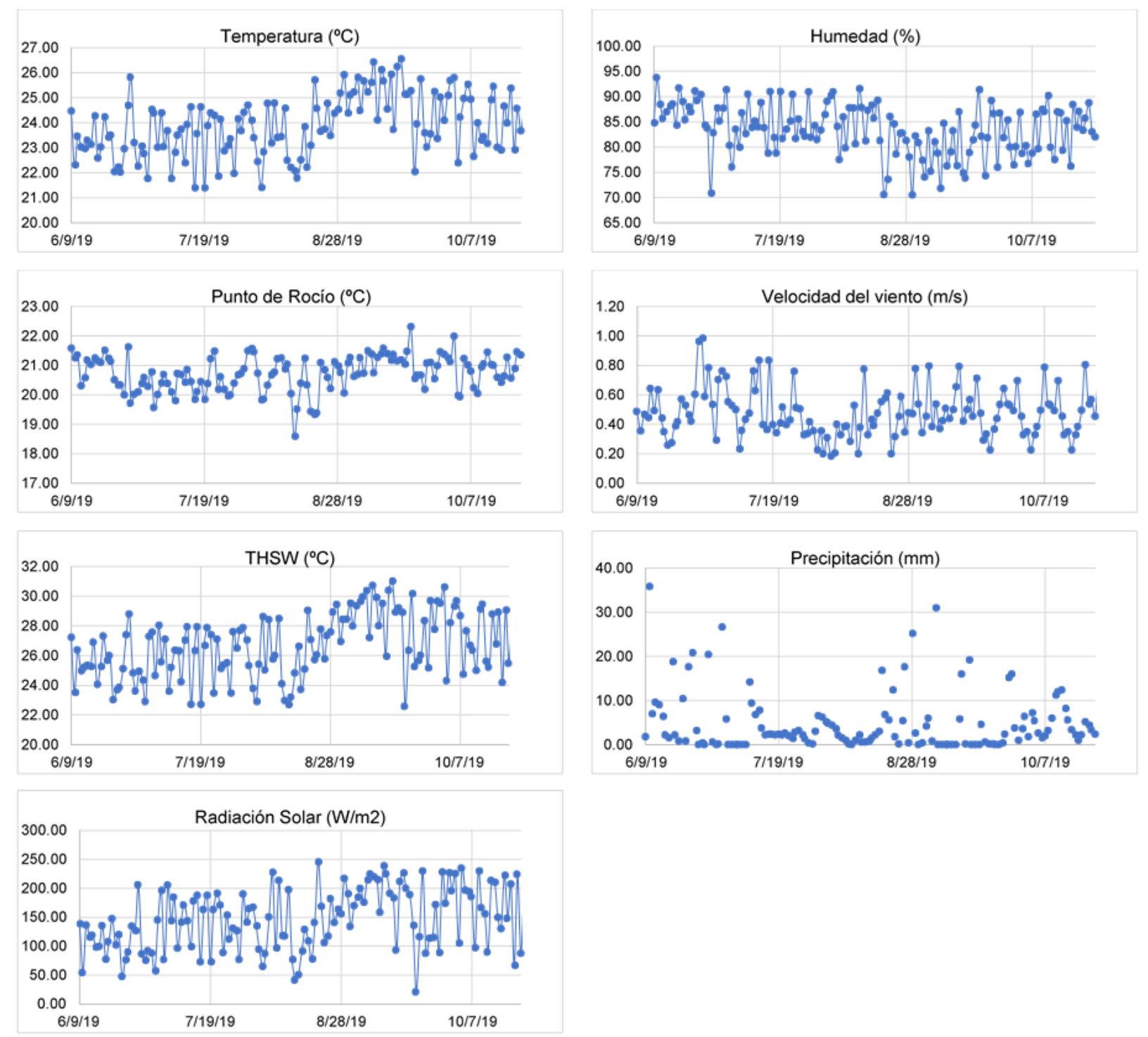

Las principales características químicas y físicas de los suelos donde se encontraron las parcelas experimentales se muestran en el Cuadro 1. Los suelos fueron ácidos y presentaron baja fertilidad por limitado aporte de nutrientes, características habitualmente encontradas bajo condiciones tropicales. 
Cuadro 1: Características químicas y físicas del suelo

\begin{tabular}{|c|c|c|c|}
\hline Item & Bloque 1 & Bloque 2 & Bloque 3 \\
\hline $\mathrm{pH}$ & 4.71 & 4.73 & 4.67 \\
\hline $\mathrm{CE}, \mathrm{dS} / \mathrm{m}$ & 0.07 & 0.06 & 0.06 \\
\hline Capacidad de campo, \% & 18.5 & 15.8 & 13.8 \\
\hline $\begin{array}{l}\text { Punto de marchitez } \\
\text { permanente, } \%\end{array}$ & 9.25 & 7.90 & 6.91 \\
\hline Densidad aparente, $\mathrm{g} / \mathrm{cc}$ & 1.51 & 1.46 & 1.44 \\
\hline Carbono orgánico, \% & 1.02 & 0.88 & 0.62 \\
\hline Materia orgánica, \% & 1.76 & 1.52 & 1.07 \\
\hline Arena, \% & 68 & 60 & 50 \\
\hline Limo, \% & 12 & 16 & 18 \\
\hline Arcilla, \% & 20 & 24 & 32 \\
\hline Textura & $\begin{array}{c}\text { Franco - arcillo - } \\
\text { arenoso }\end{array}$ & $\begin{array}{c}\text { Franco - arcillo - } \\
\text { arenoso }\end{array}$ & $\begin{array}{c}\text { Franco - arcillo - } \\
\text { arenoso }\end{array}$ \\
\hline $\begin{array}{l}\text { Potasio intercambiable, } \\
(\mathrm{mg} / \mathrm{kg})\end{array}$ & 23.4 & 23.4 & 15.6 \\
\hline $\begin{array}{l}\text { Calcio intercambiable, } \\
\mathrm{mg} / \mathrm{kg}\end{array}$ & 274 & 130 & 132 \\
\hline $\begin{array}{l}\text { Magnesio intercambiable, } \\
\mathrm{mg} / \mathrm{kg}\end{array}$ & 24.0 & 16.8 & 18.1 \\
\hline $\begin{array}{l}\text { Sodio intercambiable, } \\
\mathrm{mg} / \mathrm{kg}\end{array}$ & 23.2 & 18.4 & 16.1 \\
\hline $\begin{array}{l}\text { Acidez intercambiable, } \\
\mathrm{mg} / \mathrm{kg}\end{array}$ & 218 & 219 & 172 \\
\hline Hierro, $\mathrm{mg} / \mathrm{kg}$ & 305 & 358 & 459 \\
\hline Manganeso, mg/kg & 9.6 & 6.0 & 3.3 \\
\hline Cobre, mg/kg & 1.1 & 0.75 & 0.53 \\
\hline Zinc, mg/kg & 0.7 & 0.4 & 0.4 \\
\hline Boro, mg/kg & 0.11 & 0.17 & 0.13 \\
\hline Fósforo, $\mathrm{mg} / \mathrm{kg}$ & 7.6 & 3.7 & 5.5 \\
\hline Azufre, mg/kg & 7.8 & 9.6 & 3.6 \\
\hline CICE, meq/100g & 3.64 & 3.35 & 2.82 \\
\hline
\end{tabular}

$\mathrm{pH}=$ potencial de hidrogeniones; $\mathrm{CE}=$ conductividad eléctrica; $\mathrm{mg}=$ miligramos; $\mathrm{kg}=$ kilogramos; $\mathrm{CICE}=$ capacidad de intercambio catiónico.

\section{Fases de desarrollo}

Los genotipos evaluados tuvieron diferencias significativas en todas las variables de desarrollo $(P<0.05)$, a excepción del tiempo de secado de aquenios y del porcentaje de semillas rudimentarias $(P>0.05)$ (Cuadro 2). El genotipo 1 fue el material que tomó mayor tiempo (140.1 días) en la sumatoria de las etapas de crecimiento y desarrollo (fase de floración) y el genotipo 4 fue el que presentó menores tiempos (127.2 días) con 
diferencias significativas $(P<0.001)$. Con relación a la producción de semilla sexual, la fertilización tuvo efecto $(P<0.001)$ al generar 2.14 veces más semillas, y en general incrementó el tiempo de las fases evaluadas en aproximadamente 5 días. Los genotipos 5 y 7 fueron los materiales con mayor producción de cabezuelas por plantas, mayor porcentaje de semillas llenas y presentaron el número más alto de semillas por planta, esto asociado probablemente a su mayor número de ramas. De igual forma se destaca el alto porcentaje de semillas vacías en todos los genotipos, así como de semillas rudimentarias, que determinan que más del $30 \%$ de las semillas no tienen viabilidad física.

\section{Germinación}

En el Cuadro 3 se presenta el porcentaje de germinación de la semilla sexual de los genotipos evaluados con los tres tratamientos pre-germinativos usados. De acuerdo con los resultados obtenidos hubo diferencias significativas entre genotipos y tratamientos $(P=0.0001)$. En promedio los porcentajes de germinación fueron 46.87, 53.53 y 25.97 para el tratamiento testigo, uso de agua a $80^{\circ} \mathrm{C}$ y ácido sulfúrico al $50 \%$, respectivamente, y este proceso inició a los tres días después de la siembra.

La germinación de las semillas sin tratamiento fue significativamente menor en el genotipo 1 frente a los otros 6 genotipos $(P<0.0001)$. Además, este mismo material tuvo los valores más bajos cuando se usó el tratamiento con agua y de ácido sulfúrico, aunque no tuvo diferencias significativas con los genotipos 2 y 7 . La fertilización incrementó en promedio $9.2 \%$ la germinación de los diferentes genotipos y el tratamiento que logró mayor germinación fue el uso de agua a $80^{\circ} \mathrm{C}$ y el tratamiento con menor germinación fue el que utilizó ácido sulfúrico. En general los genotipos 3 y 6 fueron los que presentaron mayor porcentaje de germinación, pero solo tuvieron diferencias significativas frente al genotipo 1 .

\section{Discusión}

Conocer la fenología de las especies arbustivas y su potencial de propagación permiten no solo lograr un mayor aprovechamiento, sino también un uso más eficiente y económico. Los resultados de los tiempos de cada una de las fases evaluadas coinciden con lo reportado por Saavedra ${ }^{(23)}$. En general los tiempos de crecimiento y desarrollo fueron modificados por la fertilización, debido probablemente a la disminución de algunos factores de estrés. Un uso adecuado de fertilización favorece la producción de semilla y genera un mejor desarrollo ${ }^{(24)}$. Saavedra ${ }^{(23)}$ al evaluar tres orígenes de $T$. diversifolia, encontró una duración del proceso de desarrollo muy similar entre los materiales, con una duración del desarrollo vegetativo entre 88 y 137 días, el proceso de floración entre 18 y 22 días, y una formación de frutos entre 13 y 18 días. En cuanto a las semillas llenas, vacías y rudimentarias, esta misma autora encontró valores entre $65 \mathrm{y}$ $75 \% ; 15.75$ a $26 \%$ y 9.31 a $12 \%$, respectivamente, similares a las del presente estudio. 
La producción de semilla para $T$. diversifolia también ha sido determinada por diferentes autores. En un estudio en África se encontró que esta especie puede producir entre $35 \mathrm{y}$ 212 capítulos por planta, entre 32 y 62 semillas por capítulo, entre 1,120 a 13,144 semillas por planta y que 1,000 semillas pesan entre 6.42 y $7.5 \mathrm{~g}$. Estos resultados evidencian gran variabilidad en esta especie, y los resultados concuerdan con los hallados en esta evaluación a excepción del número de semillas por capítulo, ya que en este estudio se identificó un mayor número ${ }^{(7)}$.

La producción de semilla estuvo relacionada con el número de ramas que favorece una mayor producción de botones florales. El manejo al momento del corte, como por ejemplo la altura de la poda, favorece mayor número de ramas y producción de biomasa ${ }^{(25,26)} . T$. diversifolia se puede reproducir tanto por semilla gámica como por semilla asexual, lo que le confiere gran capacidad de reproducción y colonización de nuevos hábitats ${ }^{(7,27)}$. Esta especie florece y produce semillas durante todo el año, especialmente en los meses de octubre y noviembre, aunque por condiciones ambientales puede ser anual ${ }^{(8,9)}$. Las plantas maduras producen de 80,000 a 160,000 semillas por metro cuadrado anualmente, de las cuales se desarrollan completamente el $70 \%$, pero se han reportado porcentajes de germinación por debajo del $30 \%$ en condiciones naturales ${ }^{(7,10)}$ como ocurrió en los genotipos 1 y 5 del presente estudio.

Los resultados encontrados en este estudio indican que la germinación de $T$. diversifolia durante los primeros meses de pos-cosecha es aceptable, aunque variable entre genotipos. Algunos autores destacan que esta variabilidad puede deberse a una latencia fisiológica ${ }^{(20)}$ y a irregularidades observadas en su división meiótica ${ }^{(10,13,28)}$. Existen diferencias entre ecotipos en su capacidad de germinación. Los resultados encontrados coinciden con un estudio de 29 materiales colectados en Cuba donde se hallaron diferencias significativas en los porcentajes de germinación los cuales oscilaron entre el 5 y el $67 \%{ }^{(29,30)}$. Pero los resultados se encuentran por debajo de lo reportado en otros estudios donde se alcanzaron porcentajes mayores al $70 \%$ en algunos materiales ${ }^{(17,20)}$. También, estudios realizados en China demuestran la variabilidad de la reproducción gámica de esta especie en cinco regiones de la provincia de Yunnan ${ }^{(30)}$. En aquel estudio se determinaron los mayores rangos de germinación (29.5 a $55.5 \%$ ) con temperaturas entre los 20 y $30{ }^{\circ} \mathrm{C}$. Los resultados obtenidos coinciden con los del presente estudio respecto a que, la mayor germinación ocurre entre los primeros cinco a diez días para ambas condiciones estudiadas $^{(30)}$.

Se ha identificado que aproximadamente el $65 \%$ de los granos de polen carecen de núcleos espermáticos, indicando una fertilidad cercana al $30 \%{ }^{(10)}$. De igual manera al estudiar el desarrollo de la microsporogénesis, se reconocieron anormalidades cromosómicas en $32 \%$ de células en metafase I y anafase I, identificando cromosomas rezagados, y en la metafase II, $47 \%$ de las células presentaron asincronía de un juego de cromosomas y cromosomas rezagados. Se ha observado que en algunas especies de dicotiledóneas los dos núcleos resultantes de la primera división meiótica entran asincrónicamente en la segunda ${ }^{(31)}$, lo cual podría resultar en esterilidad masculina, 
anomalía que también la han relacionado con la orientación del huso, cromosomas rezagados y puentes anafásicos que afectan la conformación de las tétradas ${ }^{(32)}$. La presencia de cromosomas rezagados en la anafase I puede deberse a la falta de tensión que las enzimas sensitivas del cinetocoro ejercen sobre las fuerzas del huso, evitando así el arrastre de los cromosomas hacia los polos, o a que al menos uno de los cromosomas esté desalineado, generando señales negativas que la célula identifica ${ }^{(13)}$.

También es importante mencionar que existen criterios divergentes sobre la viabilidad y latencia de la semilla de $T$. diversifolia. Se ha informado que el almacenamiento de la semilla y el momento de colección tienen un importante papel en la viabilidad de la semilla sexual de esta especie. Diversos autores reportaron que un almacenamiento por encima de los cuatro meses y una recolección de semilla cuando los aquenios se encuentren de color marrón puede incrementar el porcentaje de germinación hasta un $90 \%{ }^{(25,33)}$. Otros autores de igual forma reportan que procesos pre-germinativos como el ácido sulfúrico y el agua a altas temperaturas por algunos minutos $\left(80\right.$ a $\left.100{ }^{\circ} \mathrm{C}\right)$ pueden incrementar la germinación ${ }^{(16,19)}$ como los utilizados en este trabajo.

Mejorar la reproducción de esta especie vía semilla sexual incrementaría su potencial como arbustiva forrajera en los sistemas de producción pecuaria. Observaciones de campo indican que $T$. diversifolia tiene una gran capacidad para crecer clonalmente ${ }^{(6)}$, pero en la actualidad se sabe que material proveniente de semilla sexual puede favorecer el desarrollo de sistemas radiculares más extensos, plantas más vigorosas, mayor persistencia en el tiempo de los cultivos y recuperación más rápida después del corte o pastoreo, aunque aún es difícil alcanzar material seminal de buena calidad ${ }^{(11)}$.

T. diversifolia en diferentes estudios ha sido considerada como una arbustiva forrajera de alta calidad nutricional debido principalmente a sus altos contenidos de minerales (Ca y P), PC (>20\%), carbohidratos no estructurales y porcentaje de degradación (>70 \%), y a sus bajos contenidos de FDN $(<45 \%)$ y FDA $(<40 \%)^{(3,4)}$. Los valores de PC encontrados en esta especie, son tan altos o inclusive superiores a los observados en algunas leguminosas tropicales como Stylosanthes guianensis $(20 \%)^{(34)}$, Arachis pintoi $(19.7 \%)^{(35)}$ y Gliricidia sepium $(18.23 \%)^{(36)}$, y son muy superiores a los observados en la mayoría de las gramíneas tropicales, como Urochloa brizantha $(9.3 \%)^{(3)}$ y Cynodon plectostachyus $(9.23 \%)^{(37)}$. Además, la FDN y la FDA son menores que los valores comunes observados para forrajes tropicales ${ }^{(38)}$, aspecto que probablemente no limita el consumo voluntario, la degradabilidad de los nutrientes y su potencial aprovechamiento por parte de los animales ${ }^{(1,3,4)}$.

Por otro lado, el consumo de $T$. diversifolia se ha visto asociado a incrementos de la productividad animal y de la capacidad de carga en los sistemas. En Colombia se evaluó el efecto de esta arbustiva bajo condiciones de pastoreo en la producción y calidad de la leche bovina y se halló que su consumo tuvo efectos significativos en la producción de leche observándose 9.70 y $15.4 \mathrm{~kg}$ leche/ha/día, respectivamente. Además, la producción de proteína, grasa y solidos totales también fue mayor cuando los animales consumieron 
T. diversifolia $(P<0.05)^{(39)}$. Finalmente, el incremento productivo que se ha obtenido en sistemas con $T$. diversifolia favorece menores intensidades de emisión al disminuir las emisiones de $\mathrm{CH}_{4}$ por fermentación entérica. Los resultados no encontraron diferencias en las emisiones diarias entre dietas convencionales y dietas con $25 \%$ de inclusión de $T$. diversifolia $(P=0.351)$; sin embargo, identificaron diferencias en las emisiones por $\mathrm{kg}$ de peso ganado en animales en ceba $(P=0.002)$ al pasar de $22.3 \mathrm{~kg} \mathrm{de} \mathrm{CO}_{2}$-eq $/ \mathrm{kg}$ de peso ganado en la dieta con brachiarias a $14.9 \mathrm{~kg}$ de $\mathrm{CO}_{2}$-eq/ $/ \mathrm{kg}$ de peso cuando los animales tuvieron acceso a T. diversifolia ${ }^{(40)}$.

\section{Conclusiones e implicaciones}

T. diversifolia tiene genotipos con fases de crecimiento y desarrollo significativamente diferentes entre ellos, modificando su momento reproductivo y de producción de semilla sexual. Según los resultados encontrados, existen genotipos con una mayor producción de semilla sexual viable por planta como los genotipos 5 y 7 evaluados en este estudio, los cuales se pueden perfilar como aquellos más adecuados para mejorar la propagación de esta especie. Si bien esta especie tiene una germinación baja $(<50 \%)$, existen procesos pre-germinativos con la capacidad de incrementar el porcentaje de germinación como el uso de agua a $80{ }^{\circ} \mathrm{C}$ en un $15 \%$. También el uso de fertilizante aumenta no solo la producción de semilla viable sino también su germinación, razón por la cual puede ser una alternativa viable para tratar plantas productoras de semilla (20\% más de germinación). Finalmente, este estudio confirma que la especie $T$. diversifolia, posee un alto porcentaje de semillas no viables, aspecto que invita a desarrollar trabajos orientados a entender los factores responsables de este fenómeno con el fin de mejorar y facilitar su utilización, especialmente la de los genotipos destacados identificados en este trabajo, ya que esta especie ofrece un alto potencial en la alimentación animal producto de su amplia adaptación a diferentes condiciones edafoclimáticas, oferta de altos valores de PC (>20\%), bajo contenido de FDA y FDN (<20 y $40 \%$, respetivamente), presencia de diferentes compuestos secundarios que modifican la eficiencia fermentativa a nivel del rumen, y una alta degradación que puede estar por encima del $70 \%$.

\section{Agradecimientos}

Al proyecto Ganadería Colombiana Sostenible, financiado por Gobierno del Reino Unido y el Fondo Mundial para el Medio Ambiente, por aportar recursos necesarios para las evaluaciones y recolección de materiales. También, al predio El Porvenir por permitir llevar a cabo las evaluaciones en sus instalaciones, así como a Miniciencias en su convocatoria de Doctorados Nacionales 727 de 2015. 


\section{Literatura citada:}

1. Barahona R, Sánchez S. Limitaciones físicas y químicas de la digestibilidad de pastos tropicales y estrategias para aumentarla. Cienc Tecnol Agropec 2005;6(1):69-82. https://dialnet.unirioja.es/servlet/articulo?codigo $=5624715$.

2. Schultze-Kraft R, Rao IM, Peters M, Clements RJ, Bai C, Liu G. Tropical forage legumes for environmental benefits: An overview. Trop Grassl 2018;6(1):1-14. http://www.tropicalgrasslands.info/index.php/tgft/article/view/394/241.

3. Ribeiro RS, Terry SA, Sacramento JP, Silveira S, Bento CB, Silva EF, et al. Tithonia diversifolia as a supplementary feed for dairy cows. PloS One 11 2016; e0165751. https://www.ncbi.nlm.nih.gov/pmc/articles/PMC5132235/

4. Mauricio RM, Calsavara LHF, Ribeiro RS, Pereira LGR, Freitas DS, Paciullo DS, et al. Feeding ruminants using Tithonia diversifolia as forage. JDVAR 2017;5(4):00146. http://medcraveonline.com/JDVAR/JDVAR-05-00146.php.

5. Rivera JE, Chará J, Gómez-Leyva JF, Ruíz TE, Barahona R. Variabilidad fenotípica y composición fitoquímica de Tithonia diversifolia A. Gray para la producción animal sostenible. Livest Res Rural Dev 2018;30, Article \#200. http://www.lrrd.org/lrrd30/12/rive30200.html .

6. Ruiz TE, Febles G, Díaz H, Achang G. Efecto de la sección y el método de plantación del tallo en el establecimiento de Tithonia diversifolia. Rev Cubana Cienc Agr 2009;43(1):91-96. https://www.redalyc.org/pdf/1930/193015398016.pdf .

7. Obukohwo E, Umar B. Seed production, germination, emergence and growth of Tithonia diversifolia (HEMSL) A. Gray as influenced by different sowing depths and soil types. Am Eurasian J Agric Environ Sci 2014;14(5):440-444. https://www.idosi.org/aejaes/jaes14(5)14/9.pdf .

8. Pérez A, Montejo I, Iglesias J, López O, Martín GJ, García DE, Milián I, Hernández A. Tithonia diversifolia (Hemsl.) A. Gray. Pastos y Forrajes 2009;32(1):1-15. http://scielo.sld.cu/scielo.php?script=sci_arttext\&pid=S0864-03942009000100001.

9. Chagas-Paula DA, Oliveira RB, Rocha BA, Da Costa FB. Ethnobotany, chemistry, and biological activities of the genus Tithonia (Asteraceae). Chem Biodivers 2012;9(2):210235.

10. Alcorcés N, Lárez A, Mayz J. Adiciones al conocimiento citogenético de Tithonia diversifolia (Hemsl.) A Gray (Asteraceae). Acta Bot Venez 2007;30(2):267-275. http://ve.scielo.org/scielo.php?script=sci_arttext\&pid=S0084-59062007000200001. 
11. Romero O, Galindo A, Murgueitio E, Calle Z. Primeras experiencias en la propagación de botón de oro (Tithonia diversifolia, Hemsl. Gray) a partir de semillas para la siembra de sistemas silvopastoriles intensivos en Colombia. Subtrop Agroecosystems 2014; 17,524-528.

https://pdfs.semanticscholar.org/3dff/1f62f0898f9df00e320078b4b8ba80864462.pdf.

12. Ruiz TE, Febles GJ, Alonso J, Crespo G, Valenciaga N. Agronomy of Tithonia diversifolia in Latin America and the Caribbean región. In: Savon LL, Gutierrez O, Febles F, editors. Mulberry, moringa and tithonia in animal feed, and other uses. Results in Latin America and the Caribbean. ICA / EDICA. La Habana, Cuba; 2017:171-20.

13. Taylor S, Scott M, Holland A. The spindle checkpoint: a quality control mechanism which ensures accurate chromosome segregation. Chromosome Res 2004;12:599-616. https://link.springer.com/article/10.1023/B:CHRO.0000036610.78380.51.

14. Rivera JE, Gómez-Leyva JF, Castaño K, Morales JG, Chará J, Barahona R. Diversidad molecular, química y morfológica en materiales de Tithonia diversifolia (hemsl.) Gray para la alimentación animal en Colombia y México. En: Chará J, et al editores. Congreso Internacional Sobre Sistemas Silvopastoriles. Manizales, Colombia. 2017:249-255.

15. Holdridge LR. Ecología basada en zonas de vida. Leslie Holdridge. IICA. San José, Costa Rica. 1986.

16. Akinola JO, Larbi A, Farinu GO, Odunsi AA. Seed treatment methods and duration effects on germination of wild sunflower. Exp Agric 2000;36:63-69.

17. Agboola DA, Idowu WF, Kadiri M. Seed germination and seedling growth of the Mexican sunflower Tithonia diversifolia (Compositae) in Nigeria, Africa. Rev Bio Trop 2005;54(2):395-402. https://www.scielo.sa.cr/scielo.php?script=sci_arttext\&pid=S003477442006000200017

18. Nasreen S, Khan MA, Uddin S. Response of sunflower to various pre-germination techniques for breaking seed dormancy. Pak J Bot 2015;47(2):413-416.

19. Muoghalu JI, Chuba DK. Seed germination and reproductive strategies of Tithonia diversifolia (Hemsl.) A. Gray and Tithonia rotundifolia (P.M.) Blake. Appl Ecol Environ Res 2005;3(1):39-46. http://citeseerx.ist.psu.edu/viewdoc/download?doi=10.1.1.541.352\&rep=rep1\&type=pd $\mathrm{f}$

20. Santos-Gally R, Muñoz M, Franco G. Efecto de la latencia sobre la germinación de Tithonia diversifolia (Asteraceae). En: Rivera J, et al, editores. Congreso internacional sobre sistemas silvopastoriles: por una producción sostenible. Libro de Actas. Editorial CIPAV, Cali. 2019:417-425. 
21. Botero J. Potencial de extracción y utilización de nutrientes de Tithonia diversifolia y su relación con el rendimiento y calidad nutricional. [tesis doctorado]. Palmira, Colombia. Universidad Nacional de Colombia; 2017. http://bdigital.unal.edu.co/56938/2/2021-Julian_Mauricio_Botero.pdf.

22. RStudio Team. RStudio Version 1.1.447: Integrated Development for R. RStudio, Inc., Boston, MA. 2019. https://rstudio.com/.

23. Saavedra S. Fenología y fisiología de semillas de botón de oro Tithonia diversifolia (Hemsl.) Gray. [Tesis maestría]. Medellín, Colombia. Universidad Nacional de Colombia 2016 http://www.bdigital.unal.edu.co/55663/1/1128408177.2016.pdf

24. Márquez F, Sánchez J, Urbano D, Dávila C. Evaluación de la frecuencia de corte y tipos de fertilización sobre tres genotipos de pasto elefante (Pennisetum purpureum). Zootec Trop 2007;25(4):253-259. http://ve.scielo.org/scielo.php?script=sci_arttext\&pid=S0798-72692007000400003.

25. Partey SY. Effect of pruning frequency and pruning heighton the biomass production of Tithonia diversifolia (Hemsl)A. Gray. Agrofor Syst 2011;83:181-187.

26. Rodríguez I, Padilla C, Ojeda M. Características de la germinación de la semilla gámica de Tithonia diversifolia (Hemsl.) Gray y su comportamiento en condiciones de vivero. Livest Res Rural Dev 2019;31, Article \#69. http://www.lrrd.org/lrrd31/5/idalma31069.html.

27. Ruíz TE, Febles G, Torres V, González J, Achan G, Sarduy L, Díaz H. Evaluación de materiales recolectados de Tithonia diversifolia (Hemsl.) Gray en la zona centrooccidental de Cuba. Rev Cubana Cienc Agríc 2010;44:291-296. https://biblat.unam.mx/hevila/Revistacubanadecienciaagricola/2010/vol44/no3/13.pdf

28. Rodríguez I, Padilla C, Torres V. Evaluación de tres métodos de poda de Tithonia diversifolia (Hemsl.) Gray bajo condiciones de pastoreo. Livest Res Rural Dev 2020; 32, Article \#73. from http://www.lrrd.org/lrrd32/5/idal32073.html.

29. Ruiz TE, Febles G, Achan G, Díaz H, González J. Capacidad germinativa de semilla gámica de materiales colectados de Tithonia diversifolia (Hemsl.) Gray en la zona centrooccidental de Cuba. Livest Res Rural Dev 2018;30, Article \#81. http://www.lrrd.org/lrrd30/5/ruiz30081.html.

30. Wang SH, Sun WB, Cheng X, Yang YM. Reproductive characteristics of Tithonia diversifolia and its geographical spread in Yunnan Province of South-West China. Acta Entomol Sin 2008;28(3). https://www.researchgate.net/publication/288293086. 
31. González F, Rudall P, Furness C. Microsporogenesis and systematics of Aristolochiaceae. Bot J Linn Soc 2001;137:221-242. https://academic.oup.com/botlinnean/article/137/3/221/2557156 .

32. Mendes-Bonato AB, Junqueira R, Pagliarini MS, Valle CB Oliveira MI. Unusual cytological patterns of microsporogenesis in Brachiaria decumbens abnormalities in spindle and defective cytokinesis causing precocious cellularization. Cell Biol Int 2002; 26

(7):641-646.

https://www.sciencedirect.com/science/article/abs/pii/S1065699502909297

33. Padilla C, Rodríguez I, Ruiz TE, Herrera M. Determinación del mejor momento de cosecha de semilla gámica, Tithonia diversifolia (Hemsl.) Gray. Livest Res Rural Dev 2018;30, Article \#71. http://www.lrrd.org/lrrd30/4/idal30071.html.

34. La OO, González H, Vásquez M C, Estrada A. Composición química, digestibilidad ruminal in situ y fraccionamiento proteico de Stylosanthes guianensis. Ciencia en la frontera: revista de ciencia y tecnología de la UACJ 2018;15(2):29-35. http://erevistas.uacj.mx/ojs/index.php/cienciafrontera/article/view/2890.

35. Khan MT, Khan NA, Bezabih M, Qureshi M S, Rahman, A. The nutritional value of peanut hay (Arachis hypogaea L.) as an alternate forage source for sheep. Trop Anim Health Prod 2013;45:849-853.

36. Silva SF, Carneiro MS, Edvan RL, Pereira ES, Neto L, Pinto AP Camilo D. Agronomic characteristics and chemical composition of Gliricidia sepium grown under different residual heights in different seasons. Cienc Investing Agrar 2017;44(1):35-42.

37. Montoya E, Barahona R, Chará J. The nutritional balance of early lactation dairy cows grazing in intensive silvopastoral systems. Ciênc Anim Bras 2017;18:1-12. https://www.scielo.br/scielo.php?script=sci_arttext\&pid=S1809-68912017000100218.

38. Souza LF, Mauricio RM, Gonçalves L C, Saliba EOS, Moreira GR. Produtividade e valor nutritivo da Brachiaria brizantha cv. Marandu em um sistema silvipastoril. Arq Bras Med Vet Zootec 2007;59(4):1029-1037. https://www.scielo.br/scielo.php?script=sci_arttext\&pid=S0102-09352007000400032.

39. Rivera JE, Cuartas CA, Naranjo JF, Tafur O, Hurtado EA, Arenas FA, Chará J, Murgueitio E. Efecto de la oferta y el consumo de Tithonia diversifolia en un sistema silvopastoril intensivo (SSPi), en la calidad y productividad de leche bovina en el piedemonte Amazónico colombiano. Livest Res Rural Dev 2015;27, article \#189. http://www.lrrd.org/lrrd27/10/rive27189.html. 
40. Molina IC, Donneys G, Montoya S, Villegas G, Rivera JE, Chará J, Barahona R. Emisiones in vivo de metano en sistemas de producción con y sin inclusión de Tithonia diversifolia. Congreso Nacional de Sistemas Silvopastoriles y Congreso Internacional de Sistemas Agroforestales. Puerto Iguazú, Misiones, Argentina, 2015:678-682. 
Cuadro 2: Fases de desarrollo agronómico y producción de semilla sexual de diferentes genotipos de Tithonia diversifolia

\begin{tabular}{|c|c|c|c|c|c|c|c|c|c|c|c|c|}
\hline \multirow{2}{*}{ Parámetro } & \multicolumn{7}{|c|}{ Tratamientos-Genotipos } & \multirow{2}{*}{$\frac{\text { Fert }}{\text { No }}$} & \multirow{2}{*}{$\begin{array}{l}\mathbf{G e} \\
\mathbf{S i}\end{array}$} & \multirow{2}{*}{$\frac{\text { Fert }}{\text { P-value }}$} & \multicolumn{2}{|c|}{ EEM } \\
\hline & 1 & 2 & 3 & 4 & 5 & 6 & 7 & & & & & \\
\hline Fase vegetativa, días & $86.8^{\mathrm{a}}$ & $84.2^{\mathrm{a}}$ & $80.6^{\mathrm{ab}}$ & $74.3^{\mathrm{c}}$ & $76.2^{\mathrm{bc}}$ & $80.7^{\mathrm{ab}}$ & $76.3^{\mathrm{bc}}$ & 77.1 & 82.4 & $<0.001$ & $<0.001$ & 0.92 \\
\hline Fase reproductiva, días & $31.8^{\mathrm{a}}$ & $29.2^{\mathrm{ab}}$ & $28.8^{\mathrm{ab}}$ & $31.5^{\mathrm{ab}}$ & $29.3^{\mathrm{ab}}$ & $27.5^{\mathrm{b}}$ & $31.3^{\mathrm{ab}}$ & 29.9 & 30 & 0.022 & 0.845 & 0.4 \\
\hline Secado de aquenios, días & 21.8 & 21.7 & 22.7 & 21.5 & 23.3 & 23.3 & 21.7 & 22.7 & 21.7 & 0.67 & 0.134 & 0.36 \\
\hline Fase de floración, días & $140^{\mathrm{a}}$ & $135^{\mathrm{ab}}$ & $132^{\mathrm{bc}}$ & $127^{\mathrm{c}}$ & $129^{\mathrm{bc}}$ & $131^{\mathrm{bc}}$ & $129^{\mathrm{bc}}$ & 130 & 134 & $<0.001$ & 0.001 & 0.91 \\
\hline Cabezuelas por planta,\# & $32.1^{\mathrm{b}}$ & $62.9^{\mathrm{a}}$ & $46.1^{\mathrm{ab}}$ & $53.6^{\mathrm{ab}}$ & $70.1^{\mathrm{a}}$ & $45.1^{\mathrm{ab}}$ & $71.9^{\mathrm{a}}$ & 36.3 & 72.7 & 0.002 & $<0.001$ & 4.14 \\
\hline Semillas por cabezuela, \# & $142^{c}$ & $155^{\mathrm{ab}}$ & $149^{\mathrm{bc}}$ & $152^{\mathrm{ab}}$ & $153^{\mathrm{ab}}$ & $156^{\mathrm{ab}}$ & $164^{\mathrm{c}}$ & 147 & 159 & $<0.001$ & $<0.001$ & 1.59 \\
\hline Semillas por planta,\# & $4,668^{\mathrm{c}}$ & $10,112^{\mathrm{ab}}$ & $6,958^{\mathrm{bc}}$ & $8,238^{\mathrm{abc}}$ & $10,908^{\mathrm{ab}}$ & $7,217^{\mathrm{bc}}$ & $11,946^{\mathrm{a}}$ & 5,460 & 11,697 & $<0.001$ & $<0.001$ & 713 \\
\hline Semillas 1lenas, $\%$ & $63.5^{\mathrm{b}}$ & $69.5^{\mathrm{a}}$ & $62.3^{\mathrm{b}}$ & $71.3^{\mathrm{a}}$ & $69.3^{\mathrm{a}}$ & $67.0^{\mathrm{ab}}$ & $62.7^{\mathrm{b}}$ & 65.5 & 67.5 & 0.025 & 0.244 & 0.91 \\
\hline Semillas vacías, $\%$ & $23.1^{\mathrm{ab}}$ & $22.8^{\mathrm{ab}}$ & $25.0^{\mathrm{a}}$ & $20.0^{\mathrm{b}}$ & $20.8^{\mathrm{b}}$ & $22.8^{\mathrm{ab}}$ & $25.7^{\mathrm{a}}$ & 21.5 & 24.3 & 0.041 & 0.006 & 0.56 \\
\hline Semillas rudimentarias, $\%$ & 13.5 & 7.67 & 12.7 & 8.83 & 9.83 & 10.2 & 11.7 & 11.1 & 10.2 & 0.059 & 0.418 & 0.56 \\
\hline
\end{tabular}

Fert= fertilización; $\mathrm{Ge}=$ genotipos, EEM= error estándar de la media

${ }^{a b c}$ Letras diferentes en una misma fila denotan diferencia $(P<0.05)$.

Cuadro 3: Porcentaje de germinación de la semilla sexual de diferentes genotipos de Tithonia diversifolia

\begin{tabular}{|c|c|c|c|c|c|c|c|c|c|c|c|c|}
\hline \multirow{2}{*}{$\mathbf{T r}$} & \multicolumn{7}{|c|}{ Genotipos (Ge) } & \multicolumn{2}{|l|}{ Fert } & Ge & \multirow[t]{2}{*}{ Fert } & \multirow{2}{*}{ EEM } \\
\hline & 1 & 2 & 3 & 4 & 5 & 6 & 7 & No & Si & p-value & & \\
\hline 1 & $32.6^{\mathrm{b}}$ & $45.4^{\mathrm{a}}$ & $54.1^{\mathrm{a}}$ & $45.8^{\mathrm{a}}$ & $47.7^{\mathrm{a}}$ & $53.9^{\mathrm{a}}$ & $48.6^{\mathrm{a}}$ & 42.6 & 51.1 & 0.001 & 0.002 & 1.52 \\
\hline 2 & $39.5^{b}$ & $51.6^{\mathrm{a}}$ & $58.2^{\mathrm{a}}$ & $54.1^{\mathrm{a}}$ & $52.9^{\mathrm{a}}$ & $62.2^{\mathrm{a}}$ & $56.9^{\mathrm{a}}$ & 46.9 & 59.7 & 0.001 & 0.001 & 1.89 \\
\hline 3 & $17.5^{\mathrm{c}}$ & $22.3^{\mathrm{abc}}$ & $31.9^{\mathrm{a}}$ & $30.3^{a b}$ & $19.7^{\mathrm{c}}$ & $31.8^{\mathrm{a}}$ & $26.9^{a b c}$ & 23.7 & 30.2 & 0.001 & 0.025 & 1.31 \\
\hline
\end{tabular}

